


\title{
Fluctuation-Induced Structural Forces in Nematic Films
}

\author{
P. Ziherl, S. Žumer, and R. Podgornik \\ J. Stefan Institute, Jamova 39, 1000 Ljubljana, Slovenia and \\ Department of Physics, University of Ljubljana, Jadranska 19, 1000 Ljubljana, Slovenia
}

Received 29 October, 1998

In a brief review of the theory of pseudo-Casimir interaction in nematic liquid crystals, we describe long- and short-range fluctuation-induced forces in these materials and discuss the role of the anchoring strength in this context. We also analyze the pretransitional behavior of the interaction in the vicinity of the clearing point.

PACS numbers: 61.30.-v, 61.30.Gd, 61.30.Cz

\section{Introduction}

The discovery of polymer dispersed liquid crystals in the eighties [1] has put the liquid crystal display technology in a new perspective. Since then, the classical design of a display cell based on a liquid-crystalline material sandwiched between two transparent electrodes has been complemented by a device consisting of a composite material, e.g., a dispersion of micron-size liquidcrystalline droplets in a solid polymer matrix. The undisputed advantages of this concept in some electrooptical applications have boosted the research in the field of microconfined liquid crystals, which has evolved into one of the most vivid branches of the liquid crystal science.

The interest in the physics of severely confined systems has raised a number of both experimental and theoretical issues which did not seem very important in the past. One of them has to do with the mechanical stability of such systems, which is invariably related to the structural interaction mediated by the liquid-crystalline material. Were liquid crystals perfect, disordered liquids, they would give rise to the van der Waals force only. But they are orientationally ordered rather than structureless, and this results in an additional interaction between the walls of the host medium, which is induced by the liquid crystal itself.

The experimental studies of the structural force in liquid crystals are typically carried out by a surface force apparatus [2, 3, 4, 5], and in general they have been found to be consistent with the mean-field model of the interaction. However, there are also indications that the mean-field description of the systems studied may not be entirely adequate and that the fluctuationinduced force could play a detectable role [5].

Until the beginning of this decade, there was no explicit interest in fluctuation-induced force between solid objects immersed in liquid crystals, although its mechanism - the Casimir effect - has been well-known for half a century [6]. In the first theoretical study of the phenomenon, the long-range force due to thermal fluctuations of the order parameter in nematic, smectic, and columnar phase has been calculated [7, 8], and these results constitute the skeleton of the theory of the pseudo-Casimir interaction in liquid crystals.

In this paper, we concentrate on the fluctuationinduced force in the nematic phase and in the vicinity of the clearing point. First we discuss the dominant long-range contribution to fluctuation-induced force (Sec. II) and the subdominant short-range contribution (Sec. III). We also describe the pretransitional behavior of the structural force at the clearing point in a thin film bounded by inert substrates. Then we address to the role of anchoring (Sec. IV) and finally we compare the fluctuation-induced force to the mean-field interaction (Sec. V). In Sec. VI we conclude the paper 
and outline some of the open problems in the field.

\section{Long-range force}

Deep in the nematic phase, the most important parameter of the orientational liquid-crystalline order is the average direction of the molecules described by the director field $\mathbf{n}(\mathbf{r})$. The energetic cost of deformation of the director field is given by the Frank elastic energy

$$
H_{V}[\mathbf{n}(\mathbf{r})]=\frac{1}{2} \int\left[K_{11}(\nabla \cdot \mathbf{n})^{2}+K_{22}(\mathbf{n} \cdot \nabla \times \mathbf{n})^{2}+K_{33}(\mathbf{n} \times \nabla \times \mathbf{n})^{2}\right] \mathrm{d} V
$$

where $K_{11}, K_{22}$ and $K_{33}$ are the splay, twist, and bend elastic constant [9]. The coupling between the orientation of material and the confining substrates is described by the anchoring energy, which is usually modeled by the Rapini-Papoular ansatz

$$
H_{S}[\mathbf{n}(\mathbf{r})]=-\frac{1}{2} \int G(\mathbf{n} \cdot \mathbf{k})^{2} \mathrm{~d} S
$$

where $G$ is the anchoring strength and $\mathbf{k}$ is the preferred orientation of the director field at the substrate, the so-called easy axis. The total Hamiltonian is, of course, a sum of the bulk and the surface contribution: $H[\mathbf{n}(\mathbf{r})]=H_{V}[\mathbf{n}(\mathbf{r})]+H_{S}[\mathbf{n}(\mathbf{r})]$.

Let us now assume that the nematic sample considered is bounded by two parallel identical plates separated by $d$ (Fig. 1). In this case the equilibrium configuration of the director field, $\mathbf{n}_{0}$, is uniform, because the preferred orientations of $\mathbf{n}$ at the two plates coincide. The mean-field free energy $F_{M F}=H\left[\mathbf{n}_{0}\right]$ is equal to 0 , which implies that the corresponding thermodynamic force defined by

$$
\mathcal{F}_{M F}=-\left(\frac{\partial F_{M F}}{\partial d}\right)_{V, S}
$$

also vanishes. This exemplifies the main property of all mean-field forces: they occur only in geometries characterized by a competition between surface-induced order and bulk order - or, more precisely, by a deformed equilibrium configuration that results from this frustration.

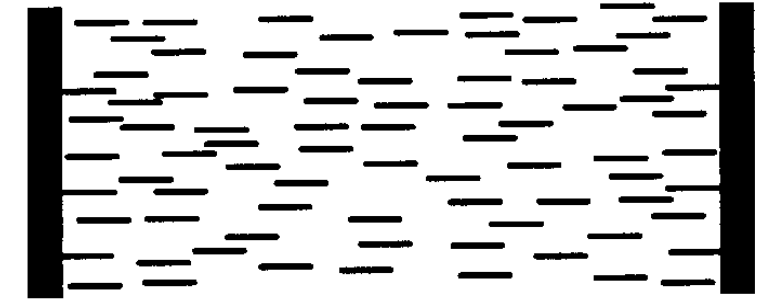

Figure 1. The system considered consists of two identical parallel plates immersed in a nematic liquid crystal.

The fluctuation-induced forces, on the other hand, are present in every confined geometry, no matter whether the equilibrium configuration of the liquidcrystalline (or any other) system is uniform or not. The fluctuation-induced forces result from the boundary conditions imposed by the substrates, which modify the spectrum of collective fluctuations. The free energy of constrained fluctuations between the substrates is different from the free energy of fluctuations in an unconstrained surrounding medium, and this gives rise to a net interaction of the substrates.

How does one calculate the interaction free energy? The free energy of the thermally excited fluctuations around the mean-field state, $\delta \mathbf{n}$, is determined by their partition function. Within the classical statistical mechanics, the partition function of the fluctuating director field is given by the functional integral over all configurations of $\delta \mathbf{n}$ that satisfy the boundary conditions

$$
\exp \left(-F_{C A S} / k T\right)=\int \mathcal{D}(\delta \mathbf{n}) \exp (-H[\delta \mathbf{n}] / k T),
$$

where $k$ is the Boltzmann constant and $T$ is the temperature [10]. The free energy consists of the bulk and surface contribution, which are proportional to the volume and the surface area of the system, respectively, 
and of the interaction free energy, which is defined simply as the residuum.

A detailed discussion of the methods of calculation of the partition function is beyond the scope of this review. At this point, we shall merely quote the result in the strong anchoring limit, which means that the fluctuations of the director field must vanish at the two plates. In this case the fluctuation-induced interaction free energy is given by

$$
F_{C A S}=-\frac{\zeta(3) k T S}{16 \pi d^{2}}\left(\frac{K_{33}}{K_{11}}+\frac{K_{33}}{K_{22}}\right),
$$

where $S$ is the area of each of the plates and $\zeta$ is the Riemann zeta function, $\zeta(3)$ being equal to $1.20205690 \ldots[7,8]$. The corresponding force reads

$$
\mathcal{F}_{C A S}=-\frac{\zeta(3) k T S}{8 \pi d^{3}}\left(\frac{K_{33}}{K_{11}}+\frac{K_{33}}{K_{22}}\right) \text {. }
$$

This attractive long-range force represents the dominant part of the pseudo-Casimir effect in nematic phase, and is completely analogous to the hightemperature limit of the electromagnetic Casimir interaction. Apart from the factor that accounts for the elastic anisotropy of the liquid crystal and clearly identified the contributions of splay-bend and twist-bend modes, the amplitude of the interaction is universal, and the Boltzmann factor witnesses the thermal origin of the force. This result was subsequently extended to account for the ruggedness of the interacting surface $[11,12]$.

\section{Short-range force}

The above discussion gives an outline of the most important manifestation of the pseudo-Casimir effect in the nematic phase, which results from director fluctuations. However, the true nematic order parameter is an irreducible second-rank tensor rather than a headless vector $\mathbf{n}$, and accomodates the degrees of order and biaxiality and the orientation of the secondary director on top of the orientation of the director itself (Fig. 2). Unlike the director modes, the three additional components of the order parameter do not arise from the broken continuous symmetry of the nematic phase and their Hamiltonians are not purely elastic.

a)

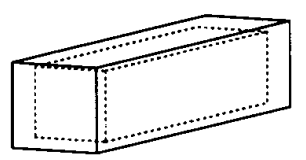

b)
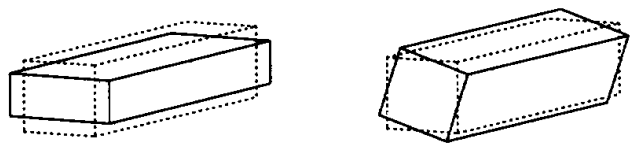

c)
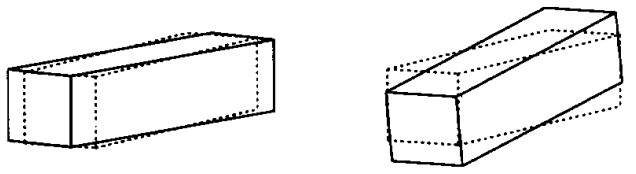

Figure 2. The five nematic degrees of freedom can be visualized by a block such that the anisotropy of its sides is proportional to the eigenvalues of the order parameter. Fluctuations of the degree of order are represented by the breathing mode (a), biaxial fluctuations modify the ratio of the short sides and rotate the block about its long axis (b), and director fluctuations correspond to the rotation of the block about the two short axes (c).

The nondirector degrees of freedom are most important in the vicinity of the nematic-isotropic phase transition, which is - at the phenomenological level - usually described by the Landau-de Gennes theory. The cornerstone of the Landau-de Gennes approach is the (truncated) Taylor expansion of the free energy density in terms of scalar invariants of the order parameter $\mathrm{Q}(\mathbf{r})$, which is usually given by

$$
h_{V}=\frac{1}{2} A\left(T-T^{*}\right) \operatorname{tr} \mathrm{Q}^{2}-\frac{1}{3} B \operatorname{tr} \mathrm{Q}^{3}+\frac{1}{4} C\left(\operatorname{tr} \mathrm{Q}^{2}\right)^{2}+\frac{1}{2} L \nabla \mathrm{Q} \vdots \nabla \mathrm{Q},
$$

where $A, B, C$, and $L$ are material constants, $T^{*}$ is the supercooling temperature. As implied by the presence of a single deformational term [which corresponds to the one-elastic-constant approximation of the Frank Hamil- tonian, Eq. (1)] the details of the liquid-crystalline elasticity are not essential in the vicinity of the phase transition. The elastic constant, $L$, is proportional to the average Frank elastic constant $K$ divided by the square 
of the degree of order. - In a similar fashion, the Rapini-Papoular anchoring energy density can be generalized to

$$
h_{S}=\frac{L}{2 \lambda} \operatorname{tr}\left(\mathrm{Q}-\mathrm{Q}_{0}\right)^{2}
$$

where $\lambda$ is the so-called extrapolation length which controls the strength of the anchoring, and $Q_{0}$ is the preferred value of the tensorial order parameter at the substrate.
In a suitable base, the above formalism reduces to the analysis of five uncoupled scalar degrees of freedom [13]. In the uniaxial nematic phase the analysis is even simpler, because the two biaxial modes are degenerate and so are the two director modes. In the one-elastic constant approximation, the generic form of the Hamiltonian that covers all five degrees of freedom [here denoted by $b(\mathbf{r})]$ reads

$$
H[b(\mathbf{r})]=\frac{L}{2}\left\{\int\left[(\nabla b)^{2}+\xi^{-2} b^{2}\right] \mathrm{d} V+\lambda^{-1} \int b^{2} \mathrm{~d} S\right\},
$$

where $\xi$ is the temperature-dependent correlation length characteristic of a particular type of fluctuations. In the case of fluctuations of the degree of order, $\xi$ is given by

$$
\xi_{N o}=\xi_{0}\left[\frac{9}{4}(1+\sqrt{1-8 \theta / 9}) \sqrt{1-8 \theta / 9}\right]^{-1 / 2}
$$

where $\xi_{0}=\sqrt{27 L C / B^{2}} \approx 10 \mathrm{~nm}$ is the bare correlation length ${ }^{1}$, and $\theta$ is the reduced temperature such that $\theta=0$ corresponds to the supercooling limit and $\theta=1$ corresponds to the phase transition temperature. The correlation length of the biaxial fluctuations reads

$$
\xi_{N b}=\xi_{0}\left[\frac{27}{4}(1+\sqrt{1-8 \theta / 9})\right]^{-1 / 2},
$$

whereas the correlation length of the director modes the broken symmetry variable of the nematic order is, of course, infinite.

This Hamiltonian also applies to director fluctuations in an external electric or magnetic field: by dividing the fluctuations into splay-bend and twist-bend modes, the vectorial Hamiltonian [Eq. 1] can be split into two uncoupled partial Hamiltonians, each operating on a scalar field. In this case, $\xi$ stands for electric or magnetic coherence length, $\sqrt{2 K / \epsilon_{a} \epsilon_{0} E^{2}}$ and $\sqrt{2 \mu_{0} K / \chi_{a} B^{2}}$ (where $K$ is the elastic constant, and $\epsilon_{a}$ and $\chi_{a}$ are the anisotropies of the dielectric permittivity and of the magnetic susceptibility, respectively) [9]. Moreover, the same Hamiltonian also describes fluctuations in the isotropic phase, where all five modes are degenerate and their correlation length is given by

$$
\xi_{I}=\xi_{0} \theta^{-1 / 2}
$$

With the Hamiltonian spelled out, we can calculate the interaction free energy. Again, we will just give the result [14] which reads

$$
F_{C A S}=\frac{k T S}{8 \pi^{2}} \int_{0}^{\infty} \ln \left(1-\left(\frac{\sqrt{\xi^{-2}+q^{2}}-\lambda^{-1}}{\sqrt{\xi^{-2}+q^{2}}+\lambda^{-1}}\right)^{2} \exp \left(-2 \sqrt{\xi^{-2}+q^{2}} d\right)\right) q \mathrm{~d} q
$$

\footnotetext{
${ }^{1}$ The meaning of the bare correlation length becomes clear as soon as $\theta=1$ is substituted in Eqs. (10) and (12): $\xi_{0}$ is the value of the correlation length of fluctuations of the degree of order in nematic and in isotropic phase at the clearing point.
} 
and is analogous to the screened van der Waals interaction between two semiinfinite dielectrics separated by an electrolyte [15]. In the following, the most important aspects of this interaction will be discussed briefly.

Let us first find out how a finite correlation length changes the fluctuation-induced interaction! Assume that the anchoring is strong, i.e., $\lambda=0$, and that $d \gg \xi$. In this case, the fluctuation-induced force is short-range:

$$
\mathcal{F}_{C A S}=-\frac{k T S}{4 \pi \xi^{2} d} \exp (-2 d / \xi),
$$

which corresponds to the interaction induced by the three non-director modes in the nematic phase (and also by the director modes in an external aligning field) and by the fluctuations in the isotropic phase. The other limit, $d \ll \xi$, recovers either splay-bend or twistbend mode contribution to the long-range interaction [Eq. (6)] within the one-elastic-constant approximation, $-\zeta(3) k T S / 8 \pi d^{3}$.

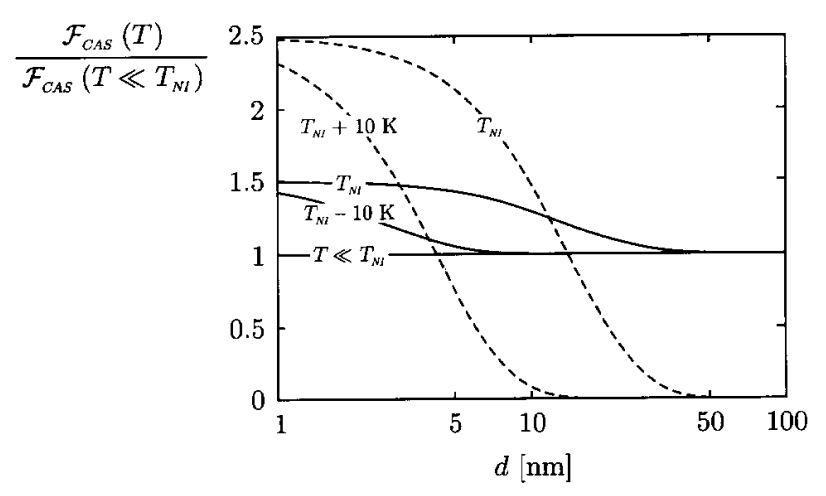

Figure 3. Pretransitional behavior of the fluctuationinduced force in a nematic liquid crystal is depicted most transparently by plotting the ratio of the temperaturedependent total fluctuation-induced force and the director modes' force. On approaching $T_{N I}$ from the nematic phase (solid line), the range of the short-range component of the fluctuation-induced force increases and in typical materials it reaches $\approx 10 \mathrm{~nm}$ at the clearing point. In the isotropic phase (dashed line), the fluctuation-induced force is entirely short-range and its correlation length decreases on heating.

The interaction free energy [Eq. (13)] describes the continuous crossover from long- to short-range force or vice versa, which occurs, for example, on approaching the nematic-isotropic transition. The overall pretransitional amplification of the fluctuation-induced force is mainly due the increase of the correlation length of the fluctuations of the degree of order, and is most pronounced in materials with small latent heat of the transition. The pretransitional behavior of the pseudoCasimir force can be illustrated by plotting the ratio of the total fluctuation-induced force and the long-range director modes' force which dominates deep in the nematic phase (Fig. 3). On approaching the clearing point from below, the range of the short-range component of the fluctuation-induced structural force increases and in typical materials it reaches $\approx 10 \mathrm{~nm}$ at $T_{N I}$. In the isotropic phase, the pseudo-Casimir force is entirely short-range, and its range decreases on heating.

Were the transition continuous, at the critical temperature the pseudo-Casimir force would be larger by $50 \%$ than deep in the nematic phase, because on top of the two director modes that dominate the pseudoCasimir effect in nematic liquid crystals, there would be an additional degree of freedom - the degree of order - characterized by long-range correlations. However, the nematic-isotropic transition is not continuous, and a significant temperature variation of the fluctuationinduced force could be observed only in the superheated or supercooled regime.

We must stress that the formalism presented is quite general and also applies to other phase transitions, and in most liquid-crystalline materials that exhibit smectic $\mathrm{C}$ and smectic A phase, the transition between the two is actually continuous. In such a case the phase transition is associated with the critical slowdown of the soft mode, and a clear and strong pretransitional amplification of the pseudo-Casimir force is expected.

\section{Anchoring}

Having analyzed the role of the correlation length in the fluctuation-induced interaction, we can proceed with a discussion of the effect of anchoring. The first important fact concerning the role of anchoring is quite obvious: the prefactor to the exponential in Eq. (13) is equal to 1 both for $\lambda \rightarrow 0$ and for $\lambda \rightarrow \infty$, which means that an infinitely weak anchoring gives rise to the same pseudo-Casimir force as an infinitely strong anchoring. This is no surprise, since the two limiting cases differ only in the type of the boundary conditions but not in the spectrum of fluctuations.

What about the anchoring strengths in between these two limiting cases? The effect of anchoring can 
be represented by the reduction factor defined as the ratio of the fluctuation-induced force at finite $\lambda$ and its strong-anchoring counterpart:

$$
R(d / \xi, \lambda / \xi)=\frac{\mathcal{F}_{C A S}(d, \xi, \lambda)}{\mathcal{F}_{C A S}(d, \xi, \lambda=0)} .
$$

In the case of infinite correlation length, which corresponds to director modes, $R$ depends only on the ratio $d / \lambda$, and is depicted in Fig. 4. Both for weak $(d / \lambda \ll 1)$ and strong anchoring $(d / \lambda \gg 1), R$ approaches 1 , and in between it reaches a minimum of 0.077 at $d / \lambda=1.230$. It can be shown that for $d / \lambda \gg 1, R$ is approximately given by $1-6 \lambda / d$, which implies that the finite-anchoring-strength correction to the long-range $d^{-3}$-force is proportional to $d^{-4}$.

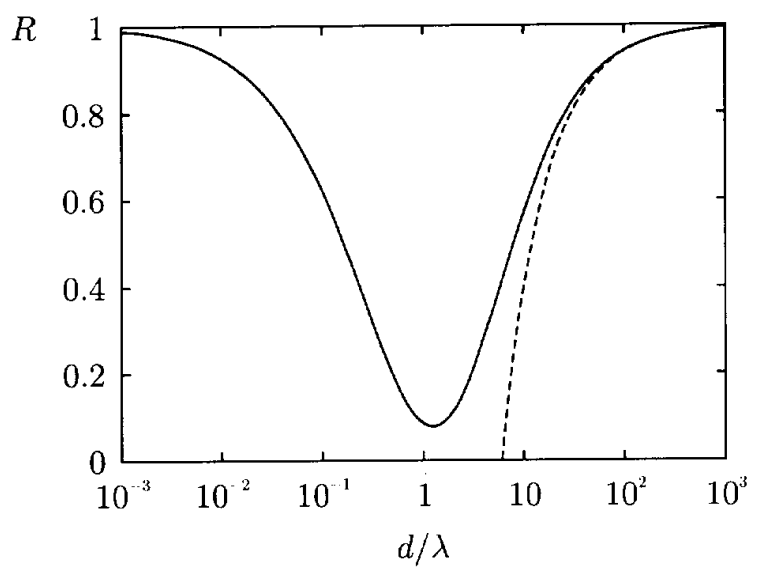

Figure 4. Reduction factor of the interaction induced by the director modes reaches a minimum of 0.077 at $d / \lambda=1.230$. The asymptotic expansion of $R$ for large $d / \lambda$ is given by $1-6 \lambda / d$ (dashed line).

If the correlation length of fluctuations is finite, $R$ depends both on $d / \xi$ and $\lambda / \xi$, and may be either a monotonic or a non-monotonic function of the interplate separation (Fig. 5). In case of rather strong anchoring, i.e., for $\lambda / \xi<1$, the reduction factor descends from 1 to a minimum and then increases to the saturated value, whereas for weak anchoring or $\lambda / \xi>1$ it decreases monotonically. As shown in Fig. 4, the saturated value of $R$ does not change if $\lambda / \xi$ is substituted by $\xi / \lambda$, and for large and small $\lambda / \xi, R(d / \xi \rightarrow \infty)$ is given by $1-4 \xi / \lambda$ and $1-4 \lambda / \xi$, respectively. The fact that $R$ saturates for large $d / \xi$ implies that asymptotically the functional form of the short-range interaction remains unchanged and that the anchoring merely reduces its magnitude. - The case $\lambda / \xi=1$ is clearly quite special, and is characterized by a reduction factor that does not level off at large $d / \xi$ 's. It can be shown that it falls off approximately as $\xi^{2} / 2 d^{2}$, so that in this particular case the fluctuation-induced force is given by $-\left(k T S / 8 \pi d^{3}\right) \exp (-2 d / \xi)$.

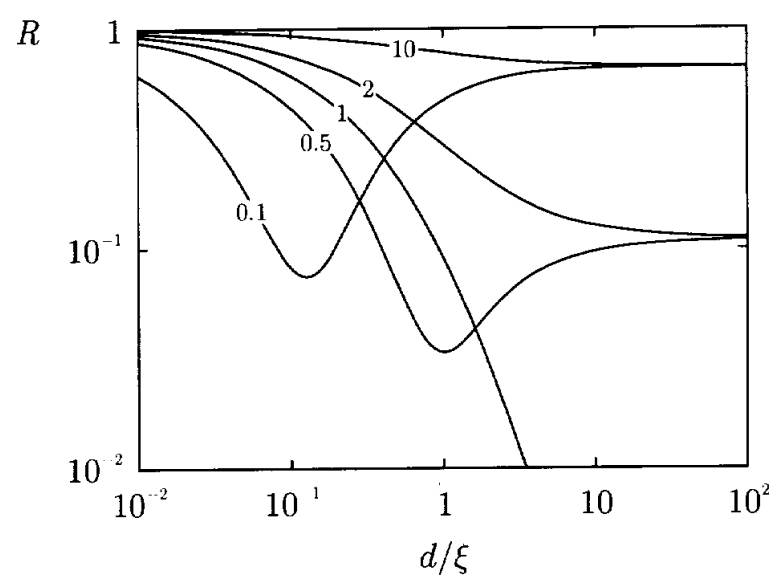

Figure 5. Reduction factor for fluctuations with finite correlation length for $\lambda / \xi=0.1,0.5,1,2$, and 10: in case of rather strong anchoring, $R$ is a nonmonotonic function of the reduced distance, $d / \xi$, and in case of rather weak anchoring $R$ decreases monotonically towards the large $d / \xi$ limit.

\section{Fluctuation-induced forces vs. mean-field forces}

It has been mentioned already that the pseudo-Casimir forces are present in any confined system, whereas the mean-field interaction only occurs in geometries characterized by deformed order parameter field. In the nematic phase, the mean-field force is largely determined by the distortion of the director induced by nonplanar confining geometry or by the hybridity of the anchoring. This interaction is repulsive and roughly proportional to $d^{-2}$, which means that it dominates in large systems. In small cavities, on the other hand, pseudoCasimir interaction should prevail since it is, as shown above, proportional to $d^{-3}$.

The basic idea behind the discussion on the relative importance of mean-field and Casimir forces relies on the notion that the director field in severely confined and irregular geometries such as (aero)gels, porous glasses, etc. is distorted because of the frustration induced by the mismatch of the easy axes across the bounding surface. Nevertheless this is usually not 
the case: below a certain void size, it may be energetically very costly to distort the director field and minimize the anchoring energy, and an undistorted liquidcrystal structure is preferred instead [16]. Uniform director configurations might be rather ubiquitous in systems with characteristic size up to, say, $100 \mathrm{~nm}$ - and since the mean-field elastic interaction is absent in uniform director configurations, the fluctuation-induced stuctural forces are probably more important than it seemed at first.

This argument brings us to another interesting aspect of the nematic order in highly constrained liquid crystals. In the vicinity of the nematic-isotropic phase transition temperature, the system can avoid the energetic penalty of an unfavorable director orientation at the wall by reducing the degree of order in the subsurface layer. In other words, the deformation of the director field is replaced by the deformation of the profile of the degree of order. This mechanism, which is expected to be most prominent in systems characterized by large anchoring energy, immediately raises the question of fluctuation-induced interaction in heterophase liquidcrystalline structures. In a forthcoming paper, we will address this aspect of the pseudo-Casimir effect by analyzing the phenomenon in systems characterized by a surface-induced degree of order substantially different from the degree of order in the bulk of the sample [17].

\section{Conclusions}

We have discussed several theoretical aspects of the fluctuation-induced interaction in nematic films and pointed to some of the systems where this interaction is expected to represent an important if not the dominant part of the structural force.

The theory of the pseudo-Casimir force in liquid crystals extends well beyond the topics described in this paper and is still, of course, far from being complete. Some of the most interesting issues in the field, such as fluctuation-induced interaction in prenematic and pres- mectic systems, distorted nematic structures, polymer liquid crystals, etc., will be addressed in the future.

\section{Acknowledgments}

We acknowledge support from Ministry of Science and Technology of Slovenia (project No. J1-0595-155498) and European Commission (INCO-Copernicus project No. IC15CT96-0744).

\section{References}

[1] J. W. Doane, N. S. Vaz, B.-G. Wu, and S. Žumer, Phys. Rev. Lett. 48, 269 (1986).

[2] R. G. Horn, J. N. Israelachvili, and E. Perez, J. Phys. France 42, 39 (1981).

[3] L. Moreau, P. Richetti, and P. Barois, Phys. Rev. Lett. 73, 3556 (1994).

[4] P. Richetti, L. Moreau, P. Barois, and P. Kékicheff, Phys. Rev. E54, 1749 (1996).

[5] B. D. Swanson and L. B. Sorensen, Phys. Rev. Lett.75, 3293 (1995)

[6] H. B. G. Casimir, Proc. Kon. Ned. Akad. Wet. 51, 793 (1948).

[7] A. Ajdari, L. Peliti, and J. Prost, Phys. Rev. Lett.66, 1481 (1991).

[8] A. Ajdari, B. Duplantier, D. Hone, L. Peliti, and J. Prost, J. Phys. II France 2, 487 (1992).

[9] P. G. de Gennes and J. Prost, The physics of liquid crystals (Clarendon Press, Oxford, 1993).

[10] P. M. Chaikin and T. C. Lubensky, Principles of condensed matter physics (Cambridge University Press, Cambridge, 1995).

[11] H. Li and M. Kardar, Phys. Rev. Lett. 67, 3275 (1991).

[12] H. Li and M. Kardar, Phys. Rev. A 46, 6490 (1992).

[13] E. I. Pokrovskii and E. I. Kats, Zh. Eksp. Teor. Fiz. 73, 774 (1977) [Sov. Phys. JETP 46, 405 (1977)].

[14] P. Ziherl, R. Podgornik, and S. Žumer, Chem. Phys. Lett. 295, 99 (1998).

[15] J. Mahanty and B. W. Ninham, Dispersion forces (Academic Press, London, 1975).

[16] O. D. Lavrentovich and P. Palffy-Muhoray, Liquid Crystals Today 5, 5 (1995).

[17] P. Sheng, Phys. Rev. Lett.37, 1059 (1976). 